\title{
PINTURA DEL XIX EN CÁDIZ. ANTONIO MENSAQUE Y RAFAEL BENJUMEA
}

\author{
XIX PAINTING IN CÁDIZ. ANTONIO MENSAQUE \\ Y RAFAEL BENJUMEA
}

\author{
Fernando Pérez Mulet \\ Universidad de Cádiz, España \\ fernando.mulet@uca.es
}

\begin{abstract}
Breve contribución al conocimiento de la obra temprana de los pintores sevillanos Mensaque y Benjumea mediante la publicación de cuatro lienzos existentes en la casa que hoy es sede de la Universidad Nacional de Educación a Distancia en Cádiz.

Palabras clave: Pintura, siglo XIX, Cádiz, Antonio Mensaque, Rafael Benjumea

Contribution to the knowledge of the sevillians painters Antonio Mensaque and Rafael Benjumea with four existing unpublished tables at the house of the UNED (Universidad Nacional de Educación a Distancia) en Cádiz

Keywords: Painting, $19^{\text {th }}$ century, Cádiz, Antonio Mensaque, Rafael Benjumea.
\end{abstract}

La sede en Cádiz de la UNED, Universidad Nacional de Educación a Distancia, situada en la Plaza de San Antonio, antes denominada de la Constitución, número 2 antiguo y moderno, es un hermoso edificio neoclásico de 1808, del maestro de obras Domingo Álvarez¹, que, al día de hoy, todavía conserva determinados testimonios muebles de su anterior vida doméstica, tanto neoclásica como isabelina. Entre éstos, no siendo los únicos, hoy nos detiene la presencia de un conjunto de lienzos, cuatro en total, con que las dos paredes superiores de la caja o hueco de la principal y simple escalera de ida y vuelta se coronan. Por sus

${ }^{1}$ Entre otros vid. FALCÓN MÁRQUEZ, Teodoro: Torcuato Benjumeda y la arquitectura neoclásica en Cádiz. Cádiz, 1974. JIMÉNEZ MATA, Juan y MALO DE MOLINA, Julio: Guía de arquitectura de Cádiz. Sevilla, 1995, p. 180. LORENZO DE LA SIERRA, Juan y Lorenzo, POMAR RODIL, Pablo, MARISCAL, Miguel Ángel: Guía artística de Cádiz su provincia. Cádiz y Jerez. Sevilla, 2005, t. I, p. 143. 
dimensiones y perfiles son cuadros pensados exclusivamente para cubrir los paneles ornamentales lisos que ordenan los dos frentes, uno paralelo al tramo mayor de escalones y otro, el menor, sobre las dos macetillas y escalones de enlace entre los dos tramos finales. Frente a los mismos, pues, quedan los huecos, protegidos por barandas de forja, que comunican con el piso tercero y último de lo que, en su momento, fue vivienda. Así, para su contemplación, quedan en alto, vistos desde el arranque del tramo de la escalera, y al nivel adecuado si es desde la última planta aunque convenientemente alejados y fuera del alcance de la mano del observador, situado éste tanto en el remate de la escalera como en el corredor de dicha planta.

Sin entrar en la descripción e historia del edificio en cuestión, centrados en esta serie de cuadros, el interés de los mismos reside en tratarse, por un lado, de un testimonio de la pintura sevillana de la época, mediado el siglo diecinueve, lo que no es extraño pues tanto el prestigio de dicha escuela como la cercanía de la población y, no menos, la todavía pujanza de la burguesía gaditana hacen normal la presencia de firmas sevillanas en la ciudad. La temática, por demás, es clara señal del romanticismo popular del momento y, más concretamente, del costumbrismo que descansa en la estampa tópica del andaluz fabricada por viajeros, foráneos y, ciertamente, los propios españoles. No vamos, por tanto, a entrar en consideraciones que no harían más que reiterar lo que en otros lugares y fechas ha sido convenientemente tratado ${ }^{2}$.

Si acaso, partiendo de una somera descripción, de izquierda a derecha, la imagen de una joven con mantilla a la espalda en un interior, en el primero de los cuadros, es seguida de un bandolero, de pie y desafiante, cigarro en la mano y escopeta al hombro, en el siguiente. A ambos se suman otros dos cuadros con sendas parejas, hombre y mujer de pie en una esquina de calle, en amable conversación o requiebro en un caso y, en el otro, un bandolero sentado en un exterior, absorto contemplando a la mujer que, alzada, se encuentra a su lado. Estamos, pues, frente a uno de los temas más preciosos o tratados del momento y del lugar de lo que no es nada difícil detectar la escuela sevillana y el círculo de los Montpensier del entorno del $1850^{3}$.

En los cuatro lienzos, con un regular estado de conservación, se detectan dos manos distintas y, a la vez, próximas en el estilo. El cuadro de la joven,

${ }^{2}$ MÉNDEZ RODRÍGUEZ, Luis: La imagen de Andalucía en el siglo XIX. Sevilla, 2008. CALVO SERRALLER, CARRETE PARRONDO, LLEÓ y VALDIVIESO: Iconografia de Sevilla 1790-1868. Madrid, 1991. Imagen romántica de España. Madrid, 1981, (Catálogo de exposición). REINA PALAZÓN, Antonio: La pintura costumbrista en Andalucía. Sevilla, 1979.

${ }^{3}$ Véase VALDIVIESO, Enrique: Historia de la pintura sevillana. Sevilla, 1986, pp. 359409. VALDIVIESO GONZÁLEZ, Enrique y FERNÁNDEZ LÓPEZ, José: Pintura romántica sevillana. Sevilla, 2011. 
diferenciado por el corte del lienzo y bastidor, rectangular, situado en la pared lateral menor, es, igualmente, más inseguro en el dibujo y simple en la composición, con defectos en el trazo anatómico a la altura de los tobillos en especial, con trazos más planos y modelado más simple. Los tres restantes, de una misma mano, denotan mayor seguridad tanto en el dibujo como en la composición y en el modelado. Insinúan con mayor ambición ambientes, exteriores y, por así decir, situaciones o, aunque vagamente, cierta emoción. No obstante, el uno y los otros no desentonan entre sí y, por supuesto, parecen obedecer a un proyecto o encargo común. Obviamente, no hacen otra cosa que no sea la de reiterar lo que en estampas, cuadros y otros objetos se representa de España y, concretamente, de Andalucía como esencia de lo español.

De cualquier forma el interés de la serie reside en la observación de dos firmas, las cuales, por demás, autentican las autorías de los cuadros. Por una parte, respecto al tema de la joven, hacia el ángulo inferior izquierdo, aparece la mención de "Mensaque / ¿A? 1843". Estaríamos, por tanto, ante una obra temprana o juvenil de Antonio Mensaque, pintor por entonces de unos 20 años y discípulo de la escuela sevillana de Bellas Artes así como, junto a otros destacados costumbristas, de Joaquín Domínguez Bécquer. Siendo lo más conocido de él su dedicación al bodegonismo y al retrato, ello al margen de otros datos biográficos, en esta primera etapa se nos revela como un aspirante más a la práctica costumbrista imperante.

De los tres temas restantes, al menos de momento, dada la situación de los mismos y el estado de conservación de la pintura, en el que representa a la pareja de joven bandolero sentado, también hacia el ángulo inferior izquierdo, aparecen la firma, lugar y fecha: "Rafael Benjumea / Sevilla 1843". La caligrafía es exacta, cursiva, bien encajada, muy elegante y fácilmente legible mientras que la fecha ofrece dificultades de lectura en una visión inmediata. No obstante hay una lógica en la misma. También se trataría de la factura de un joven, todavía alumno, pintor, lejos del que, mucho más tarde, triunfaría en Madrid, como su paisano Esquivel, en el círculo oficial de la Corte ${ }^{4}$. En todo caso ya se hace evidente una superioridad formal con respecto a la factura del cuadro firmado por Mensaque, lo cual justifica el que se le pueda considerar como pintor costumbrista. Por demás, adelanta en su factura y en la elección de los temas lo que hacia 1850 vendrá a desarrollar y que bien representan con más destreza, color y riqueza compositiva sus "Pareja de majos" y "Buñolera" de 1852.

De cualquier modo, la autenticidad de ambas firmas, Mensaque y Benjumea, descartan plenamente las normales atribuciones orales que hacían de estos

${ }^{4}$ TOVAR MARTÍN, Virginia: El palacio del Ministerio de Justicia y sus obras de arte. Madrid, 2004, pp. 216 y ss. 
cuadros obra de los Cabral Bejarano, deducción no desdeñable dados los estilos y proximidad de facturas ${ }^{5}$.

No hay de momento otra noticia sobre la cuestión y el nombre del dueño de la finca por aquel entonces tampoco arroja luces sobre la génesis y circunstancias de un más que seguro encargo. Queda claro que los cuadros formaban parte de la finca en ese momento, pensados en sus dimensiones y perfiles, como queda dicho, para el lugar. También es evidente que la elección de la temática es de índole doméstica, grata y universal, producto de la moda y de los gustos de la época y, por tanto, no habría que buscar otras consideraciones.

Siendo, pues, de 1843, en la fecha moraban la finca el matrimonio formado por Francisco Ximeno Harmony, gallego de Villagarcía, e Isidra Carrera, vecinos desde antiguo de Cádiz, donde casaron en 1826. De Francisco Ximeno hay referencias muy tempranas que nos lo sitúan como marino y mercader desde 1810, socio más adelante, además, de una poderosa compañía mercantil gaditana, Carreras y Cía., así como de su propia empresa, Harmony y cía., con corresponsales, sus hermanos y sobrinos, en Nueva York, y que funcionaría incluso tras su muerte hasta 1866 según se desprende de las guías comerciales gaditanas. Fallece en 1850 , sin descendencia directa, legando, de sus bienes, entre otros, la finca a su viuda, la cual continuará residiendo en la misma y que, más tarde, también legará a otros sobrinos 6 .

Será, pues, ya en 1923, cuando la propiedad de la finca pase a otras manos pero, prácticamente, conservando los mismos enseres que fueron formando parte del ajuar y mobiliario de la misma, hasta su definitiva compra por parte de la Diputación Provincial de Cádiz, propietaria pues de los cuadros, para sede de la actual delegación de la UNED.

Fecha de recepción: 30 de septiembre de 2014

Fecha de aceptación: 28 de noviembre de 2014

5 PIÑANES GARCÍA-OLÍAS, Manuel: "Rafael Benjumea pintor costumbrista", Laboratorio de Arte, 7, 1994, pp. 367-378.

${ }^{6}$ AHPCa. (Archivo Histórico Provincial de Cádiz. Sección Protocolos Notariales de Cádiz), Libro 3264, 1850, fols. 1163-1198. 


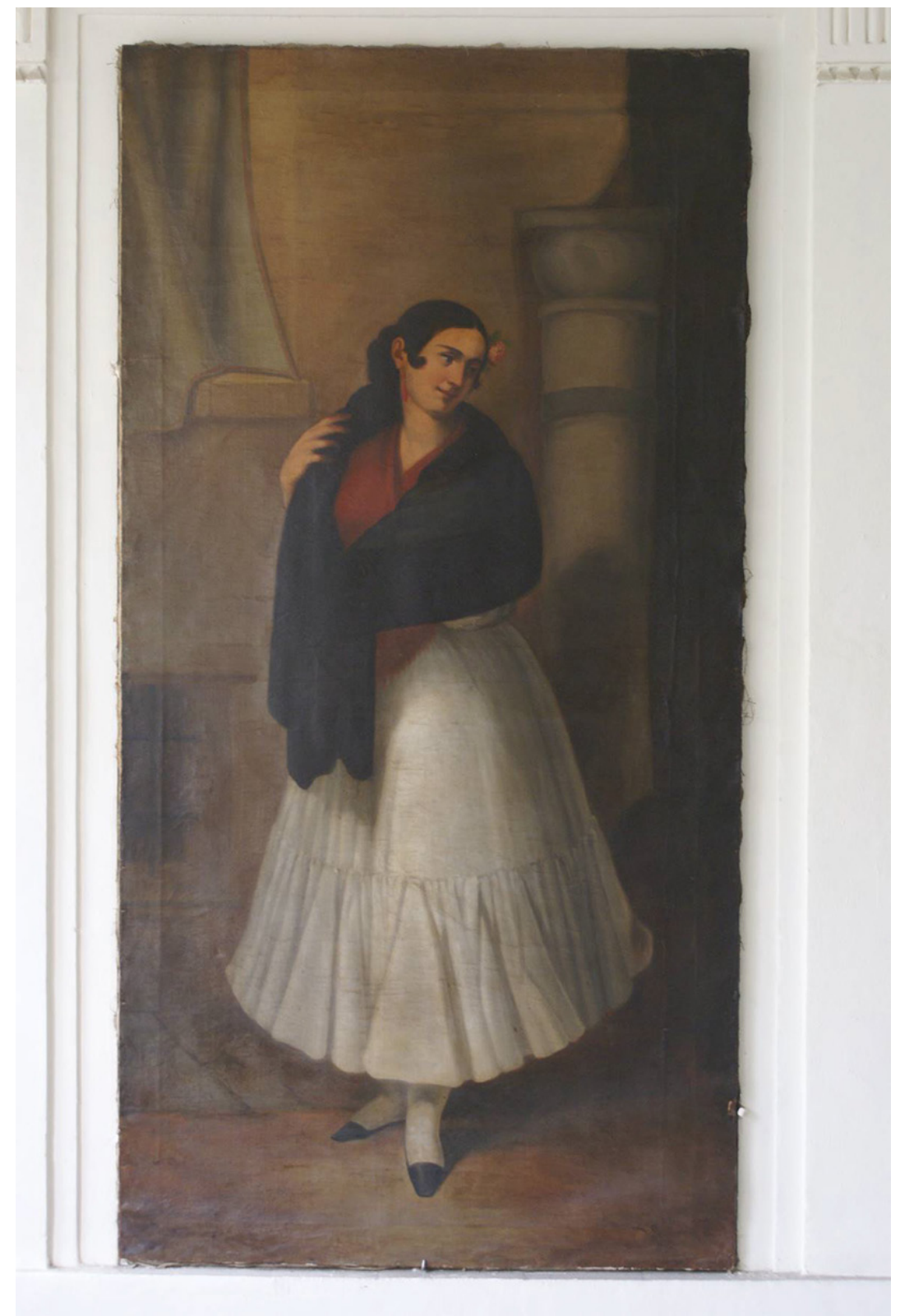

Figura 1. Joven andaluza. Antonio Mensaque. 1843.

Óleo sobre lienzo (o/l). 2,10 x 1,10 m. 


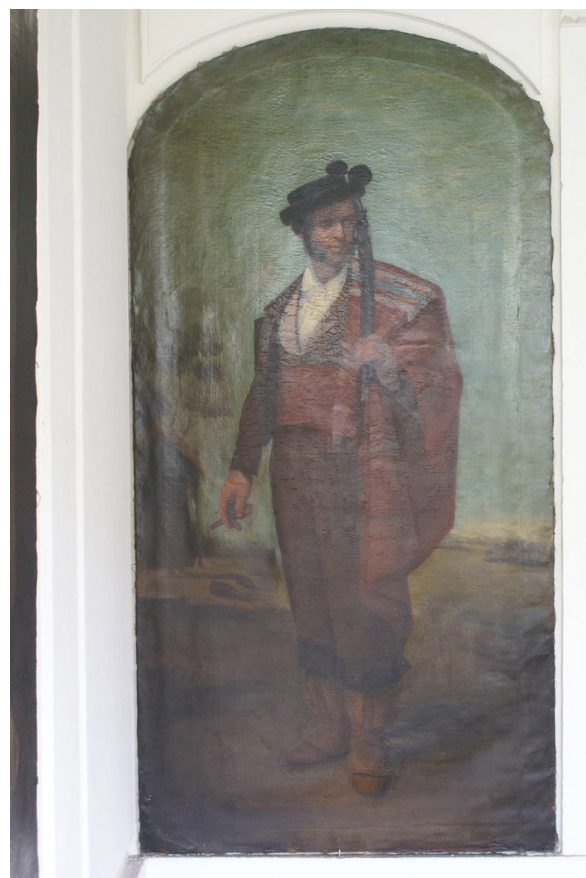

Figura 2. Bandolero. Rafael Benjumea. 1843 o/l. 2,00 x $1,00 \mathrm{~m}$.

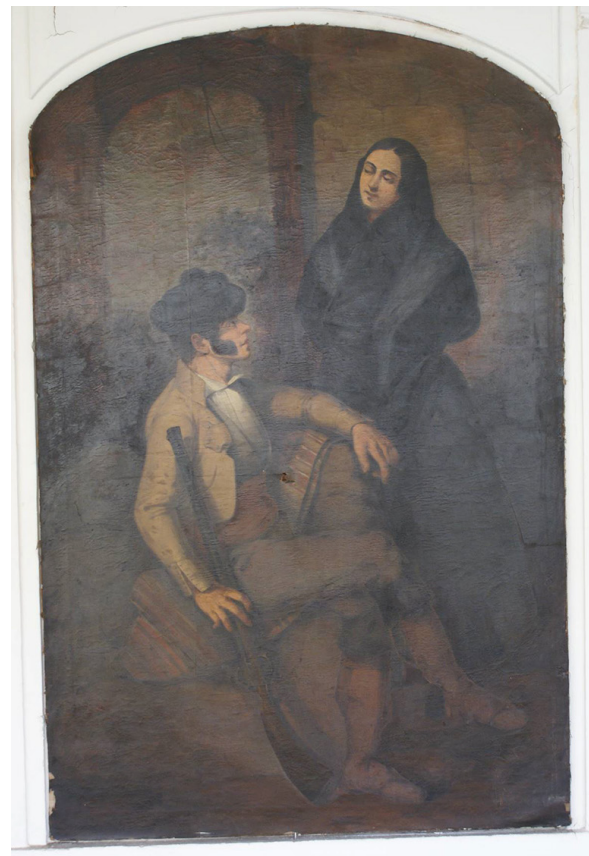

Figura 3. Bandolero y mujer conversando. Rafael Benjumea. 1843. o/l. 2,00 x 1,30 m. 


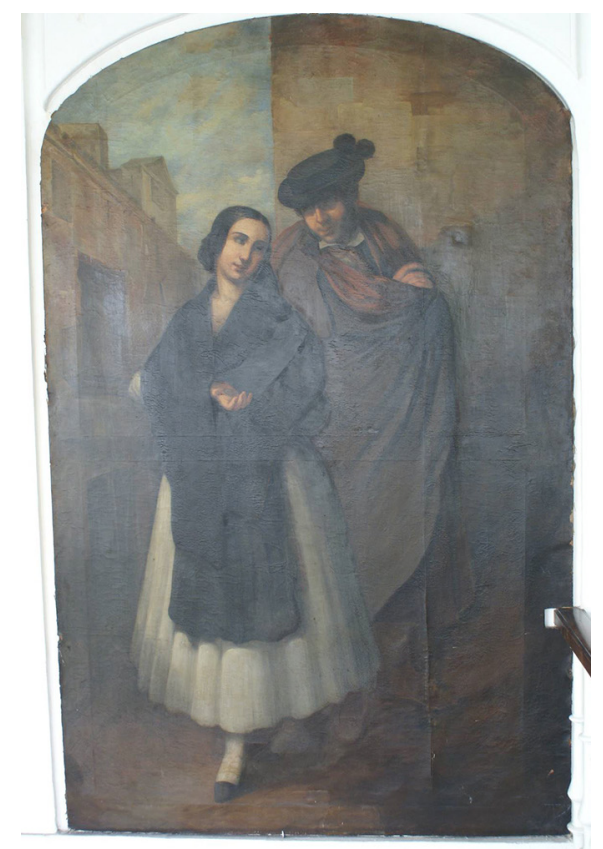

Figura 4. Pareja de majos andaluces. Rafael Benjumea. 1843. o/l. 2,00 x 1,30 m.

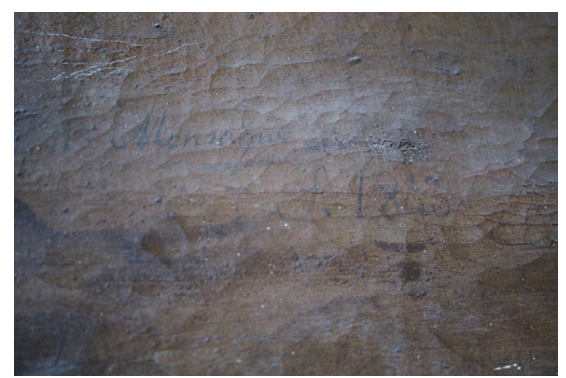

Figura 5. Joven andaluza. Detalle firma Mensaque.

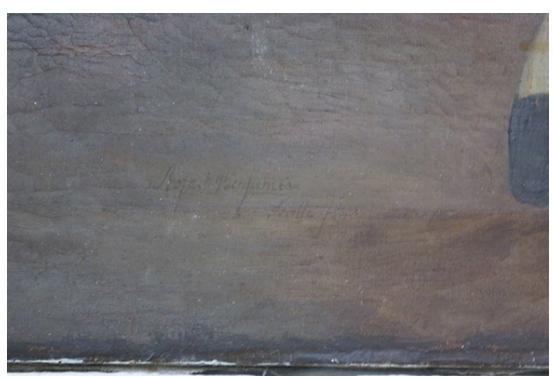

Figura 6. Pareja de majos andaluces. Detalle firma Benjumea. 